\title{
EFECTOS DEL CONTENIDO DE BORO EN LA PRODUC- CIÓN DE BULBOS Y LA CALIDAD DE FLORES EN Tulipa gesneriana cv. Rococo.
}

\section{EFFECT OF BORON CONTENT IN BULB PRODUCTION AND FLOWER QUALITY IN Tulipa genesteriana cv. Rococo}

\author{
Bobadilla, S. ' y Chimenti, C. ${ }^{2}$ \\ 'EEA INTA Esquel, Chacabuco 513, Esquel, Chubut, CP 9200 , \\ ${ }^{2}$ IFEVA / Facultad de Agronomía (UBA), Avda. San Martín 4453, CP1417 DSE, Ciudad de \\ Buenos Aires. E-mail: bobadilla@correo.inta.gov.ar
}

\section{INTRODUCCIÓN}

Durante la etapa del forzado del tulipán para producción de flores, el crecimiento se produce a partir de las reservas presentes en el bulbo, siendo necesario solo el aporte de nitrato de calcio para evitar el "stem topple" (De Hertogh y Le Nard, 1993). Por lo tanto es de fundamental importancia que los contenidos de nutrientes de los bulbos sean tales que permitan obtener una flor de calidad. Entre ellos, el boro es mencionado por la bibliografía, como uno de los que afectan particularmente la calidad de flor en diferentes especies (Ikarashi et al 1981; Tompsett, 2002). El objetivo de este trabajo fue estudiar el efecto de la concentración de boro en la solución nutritiva sobre la producción y el contenido de $\mathrm{B}$ en bulbos de tulipán y su efecto sobre la calidad de flor producida en el cultivar Rococo.

\section{MATERIALES Y MÉTODOS}

Plantas de tulipán cultivar Rococó fueron cultivadas en macetas de polietileno de $2 \mathrm{~m}$ de largo, $0,52 \mathrm{~m}$ de ancho y $0,40 \mathrm{~m}$ de alto en un medio compuesto por arena volcánica (B disponible $<0.05$ ) con 51 plantas por maceta. Las macetas fueron ubicadas debajo de una cubierta de plástico transparente de 150 micrones para evitar el efecto de eventuales precipitaciones, que reducía la radiación incidente en un $15.6 \%$ y generaba un aumento de la temperatura promedio diaria en $1,28{ }^{\circ} \mathrm{C}$. Las plantas crecieron sin limitaciones hídricas y en adecuadas condiciones fitosanitarias. Cada maceta fue rega- da diariamente durante la etapa de crecimiento con $4000 \mathrm{~mL}$ de solución nutritiva Johnson hasta comienzo de senescencia. Dicha solución tuvo la siguiente constitución (en mi de solución madre por litro de solución): KNO3 $1 \mathrm{M}$ : 6mL:Ca(NO3) $21 \mathrm{M:} 4 \mathrm{~mL}$; NH4H2PO4 1M: 2 $\mathrm{mL}$; MgSO4.H2O 1M: $1 \mathrm{~mL}$, Micronutrientes: $1 \mathrm{~mL}$; FEEDTA: $1 \mathrm{~mL}$. Solución de micronutrientes: $\mathrm{KC} 1: 3,728 \mathrm{gL}^{-1}$; MnSO4.H2O: 0,338 $\mathrm{gL}^{-1}$; ZnSO4.7 H2O: 0,575 $\mathrm{gL}^{-1}$, CuSO4.5 H2O: $0.125 \mathrm{gL}^{-1}$; H2MoO4: 0,081 $\mathrm{gL}^{-1}$. Se utilizaron 3 tratamientos que diferían en la cantidad de boro que contenía la solución: T0 0 , T1 7,73 y T2 $1.546 \mathrm{~g}$ de ácido bórico 1-1 de solución. El diseño fue de bloques al azar con una repetición de cada tratamiento por bloque siendo la submuestra 10 plantas de cada tratamiento. La cosecha de bulbos fue realizada manualmente en enero y luego de limpiados y clasificados, se extrajeron submuestras y se analizó contenido de $\mathrm{B}$. Los bulbos fueron puestos en cámara a $21{ }^{\circ} \mathrm{C}$ hasta alcanzar el estado "G". A continuación recibieron un tratamiento en seco a $9^{\circ} \mathrm{C}$ y posteriormente fueron plantados en macetas tamaño 14 , en arena volcánica, y enraizados en cámara a $6{ }^{\circ} \mathrm{C}$ hasta que los brotes alcanzaron $2 \mathrm{~cm}$ de largo. La última etapa fue realizada en invernáculo sin calefacción. Se aplicaron riegos diariamente y una vez por semana recibían una solución con nitrato de calcio $\left(2,4 \mathrm{gL}^{-1}\right)$. Obtenidas las flores se procedió a analizar algunas de sus propiedades.

\section{RESULTADOS Y DISCUSIÓN}

Los resultados mostraron que existe una asocia- 
ción entre el contenido de boro disponible en solución y el que se encuentra a nivel de tejidos en bulbo encontrándose diferencias significativas $(\mathrm{p}=0,05)$ entre los tratamientos $\mathrm{T} 0$ y $\mathrm{T} 2$ ( 7 y $9,2 \mathrm{ppm}$ respectivamente) y TI $(20,9 \mathrm{ppm})$. Se encontraron diferencias significativas $(\mathrm{p}</=$ 0,05 ) entre los tratamientos T0 1,7 , TI 1,9 y T2 2 bulbos promedio por planta. Los resultados no mostraron diferencias significativas entre tratamientos para el peso y el tamaño de los bulbos cosechados. Al ajustar una función polinomial entre el contenido de boro disponible en el sustrato y el número de bulbos promedio producido por planta se encontró una asociación significativa $(\mathrm{p}=0,05)$ que indica que la disponibilidad de boro necesaria para la máxima producción de bulbos se encuentra en el orden de los $4 \mathrm{gL}^{-1}$ de solución (Figura 1). Las diferencias en el contenido de boro en bulbos afectaron la calidad de la flor producida a partir de los mismos. La cantidad de antocianas por $\mathrm{mm}^{2}$ encontradas en los tépalos fue significativamente $(\mathrm{p}</ 0 \quad 0,05)$ diferente entre tratamientos lo que determinó importantes diferencias en la intensidad de la coloración de los mismos. Se encontró una asociación significativa $(p=0,05)$ entre el contenido de antocianas por unidad de superficie y el contenido de boro de los bulbos a partir de los cuales se originaron las flores (Figura 2). No se encontraron diferencias significativas en otras propiedades de la flor como tamaño de tépalos, diámetro de la flor, largo de vara.

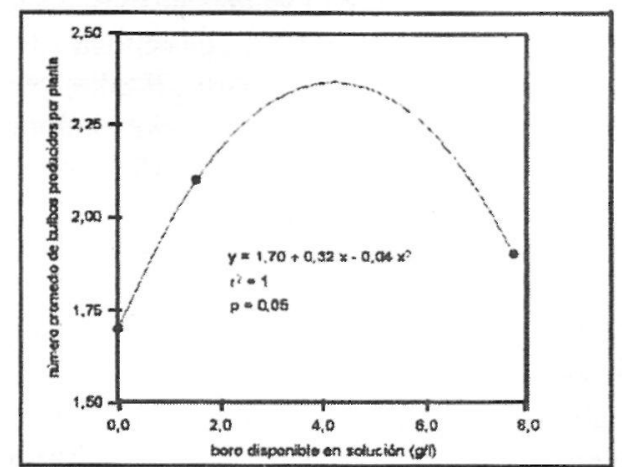

Figura 1. Relación entre el número promedio de bulbos producidos por planta $y$ el contenido de boro disponible en la solución.

Figure 1. Relationship between average number of bulbs per plant and boron available in the solution.
Los resultados demuestran que, al menos en la variedad Rococo, la producción de bulbos promedio por planta puede ser aumentada incrementando la cantidad de boro disponible para ser absorbido por la planta, y además indica el rango de valores dentro del cual se deben intensificar los estudios para determinar el valor óptimo de boro que permita alcanzar la máxima producción de bulbos. Por otro lado muestran que es posible mejorar la calidad de la flor mejorando el contenido de $\mathrm{B}$ en bulbo. La asociación encontrada indica que en el rango entre 12 y 16 ppm de $\mathrm{B}$ se encuentra la máxima concentración de antocianas y por lo tanto de coloración de las flores. Es necesario realizar experimentos dentro de este rango para determinar el valor de B por bulbo que permita alcanzar la máxima concentración de antocianas.

\section{BIBLIOGRAFÍA}

DE HERTOGH A.A.; LE NARD, M. 1993. Physiology of flower bulbs. Chapter 35 .

IKARASHI et al. 1981. The relationship between liming and "kubioremagari" (topple) or "kubiore" and "ironuke" in tulip plant on dune field. Memoirs of the Faculty of Agriculture Niigata University $\mathrm{N}^{\circ} 33$.

TOMPSETT, A. 2002. Narcissus tazzeta: Boron deficiency as a cause of flower distortion. Acta Hort. 570, ISHS 2002.

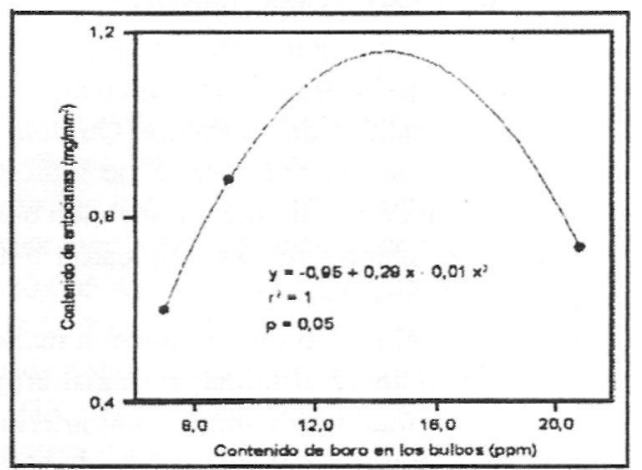

Figura 2. Relación entre contenido de antocianas

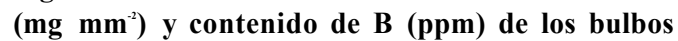
que originaron las flores.

Figure 2. Relationship between anthocyanins content ( $\left.\mathrm{mg} \mathrm{mm}^{-2}\right)$ and boron content (ppm) of the bulbs that flowered. 